\title{
"Brown Bag Clinic": A Pharmacist-led Approach to Reduce Heart Failure Hospital Readmission
}

\author{
Maggie N. Faraj ${ }^{1}$, Pharm.D., Ileana L. Piña ${ }^{2}$, MD, MPH, FAHA, FACC, Candice Garwood ${ }^{1,3}$, Pharm.D., FCCP, BCPS, BCACP
}

${ }^{1}$ Department of Pharmacy, Detroit Medical Center, Detroit, MI

${ }^{2}$ Heart Hospital, Detroit Medical Center, Detroit, MI

${ }^{3}$ Department of Pharmacy Practice, Wayne State University, Detroit, MI

\begin{abstract}
Objectives: Heart failure (HF) affects approximately 6 million in the United States and despite guideline-directed medical therapy (GDMT), still more than 20\% of patients are readmitted within 30 days. ${ }^{1,2}$ This study evaluated the impact of a "pharmacist-led HF Brown Bag Clinic" (BBC) on HF patient outcomes including readmissions and mortality.

Methods: This retrospective study, conducted at an academic medical center, included adult patients 18 to 89 years old with HF presenting to the BBC 7-14 days post HF hospitalization. Those failing to attend the BBC within 30 days of hospital discharge were in the control group. Our electronic medical records were used to capture patients' baseline characteristics and describe pharmacists' interventions. Thirty- and ninety-day post-discharge HF readmission and all-cause mortality were evaluated.

Results: A total of 32 patients met the inclusion criteria; 15 receiving intervention and 17 controls. A total of 18 HF hospital readmissions occurred, 4 (22\%) readmissions in the intervention group and $14(78 \%)$ in the control group $(p=0.06)$. Hospital readmissions within 30 days and 90 days were greater in the control group compared with the intervention group (18\% vs. $7 \%$ and $41 \%$ vs. $21 \%$ respectively). Conclusion: A pharmacist-led post-discharge clinic demonstrated numerically fewer HF hospital readmissions compared with a scheduled but "no show" control group.
\end{abstract}

Keywords: Heart failure, hospital readmissions, cardiology, pharmacist-led clinic

\section{Introduction}

As many as $20 \%$ of patients hospitalized with heart failure (HF) are readmitted within the first 30 days after discharge, constituting the largest single diagnosis of all-cause readmission. ${ }^{1,2}$ Thus, the resultant morbidity, hospitalization, and mortality pose a substantial burden on the health care and economic systems. ${ }^{3}$ At the Detroit Medical Center (DMC), the rate of $\mathrm{HF}$ hospital readmissions is similar to the national rate of $21.6 \%{ }^{4}$

A portion of these readmissions may be preventable by improvement in care transition processes and early follow-up in the post-acute setting. ${ }^{5}$ Results from recent studies have supported the positive impact that pharmacists can have on various cardiac patients such as those with $\mathrm{HF}$, coronary heart disease, or cardiovascular disease risk factors. ${ }^{6-9}$ To reduce HF hospital readmissions and improve survival at the DMC, we established the "pharmacist-led HF Brown Bag Clinic" (BBC).

The $B B C$ was designed to serve patients early after discharge to ensure successful transitions of care and to optimize medication regimens while emphasizing the importance of medication adherence and lifestyle modifications. We hypothesized that services provided in a BBC would lead to a reduction in the 30- and 90-day hospital readmission and mortality rates.

Correspondence: Maggie Faraj, Pharm.D., BCACP

Ambulatory Care Clinical Pharmacist

IHA

24 Frank Lloyd Wright Dr, Ann Arbor, MI 48105

Phone: (734) 327-6428

\section{Methods}

$B B C$ Design

The service was developed by the HF cardiologist in collaboration with a cardiology pharmacy specialist in the department of pharmacy and was embedded within an outpatient cardiology clinic at the DMC. The BBC was scheduled for two half-days per week.

The HF cardiologist provided inpatient care during hospitalization, initiated guideline-directed medical therapy (GDMT) if appropriate, and made referral to the $B B C$ post hospital discharge. Thus, appointments in the BBC were scheduled prior to a patient's discharge from the hospital, in best effort to provide transitions of care. Clinical services were delivered by a PGY-2 ambulatory care pharmacy resident supervised by the cardiology pharmacy specialist and the HF cardiologist under a collaborative agreement. Patients were asked to bring all medications to the BBC appointment in a brown bag, hence the name of the clinic. During the BBC appointment, medication reconciliation was completed, limited physical exam was performed, HF symptom history was conducted, GDMT was initiated and/or optimized according to collaborative agreement, and cardiovascular health-related patient education was provided. Any medication changes aside from GDMT addressed in the collaborative agreement, were conducted via contact with the primary care provider by telephone or letter.

All patients received non-pharmacologic interventions. All patients were educated on their disease states, rationale for the medical therapy, and the importance of dietary and medication compliance. They were also educated on how to 
respond to escalating signs and symptoms of fluid overload (such as increased shortness of breath or peripheral edema) and provided with a plan on how to respond to particular symptoms. Specifically, patients were educated that a gain of three pounds in three days or five pounds in a week would necessitate adjustment of the diuretic regimen. At the end of every clinic visit, patients were provided with medication calendars specifying the indication, direction, and the specific time to take each medication including non GDMT medications.

Patients were scheduled for follow-up appointments in the BBC based on individual status and in attempt to titrate GDMT to maximally tolerated doses. Patients were seen for follow up more frequently upon their discharge. Once they were on maximally titrated doses of GDMT and had a stable fluid status, they were scheduled for an 8 to 12-week follow-up appointment. If the patient's clinical status appeared to be worsening since discharge, they were also examined by the HF cardiologist and/or cardiology fellow.

\section{Study Design and Patient Cohort}

We conducted a retrospective study that evaluated patients between the ages of 18 to 89 years who had a diagnosis of HF and presented to the DMC from March $1^{\text {st }}, 2019$ to September $30^{\text {th }}, 2019$ with a 90-day follow-up period. BBC appointments were scheduled 7-14 days post HF hospitalization discharge. Patients attending the $\mathrm{BBC}$ were designated as the intervention group and those failing to attend within 30 days of their hospital discharge were assigned to the control group. Our study was given expedited approval by the Institutional Review Board (IRB) at the DMC and Wayne State University.

\section{Study Outcomes}

The goal of this study was to evaluate the impact of a BBC on the rate of hospital readmissions in an underserved patient population at an urban medical institution. The primary outcome was HF hospital readmissions within 30 and 90 days. The secondary outcomes were total number of $\mathrm{HF}$ readmissions, number of interventions, and 30-day and 90-day mortality.

\section{Statistical Analysis}

Student's t-test was used to analyze continuous data and chisquare test was used to analyze categorical data. A p value less than 0.05 was considered statistically significant. Analyses were performed using Microsoft Excel version 16.33.

\section{Results}

\section{Participants}

From March 1 $1^{\text {st }}, 2019$ through September $30^{\text {th }}, 2019$, a total of 32 patients met the inclusion criteria and were scheduled to follow-up in the BBC within 7-14 days post their hospital discharge. Fifteen (47\%) patients attended the BBC and 17 (53\%) patients failed to show up to clinic and were designated as the "control" group. The baseline characteristics of the two groups were similar as shown in Table 1.

\section{Study Outcomes}

The pharmacist performed 56 interventions during 29 clinic visits, with the majority occurring during the first visit (61\%). Details of intervention are illustrated in Table 2. The most common pharmacological interventions were GDMT initiation if not done inpatient and GDMT optimization (57\%). Beta blockers were the most frequently optimized medications which resulted in a decrease in resting heart rate on subsequent clinic visits. Smoking cessation was also a common intervention.

The outcomes of HF hospital readmission are shown in Table 3. Thirty and 90-day readmission rates were statistically similar in the intervention group attending the $\mathrm{BBC}$ compared with controls but were numerically fewer in the BBC group (7\% vs $18 \%, p=0.12$ and $21 \%$ vs $41 \%, p=0.20$, respectively). There were no occurrences of 30 or 90 day mortality in either BBC or control groups. Patients who did not follow-up at the BBC had a trend towards greater $\mathrm{HF}$ exacerbations leading to recurrent $\mathrm{HF}$ readmissions with a total of 14 hospital readmissions occurring in the control group, compared with 4 in the intervention group $(p=0.06)$. One BBC participant had 4 admissions post $B B C$ appointment but was excluded from the primary outcome's analysis (30- and 90-day readmissions) due to diagnosis of hepatocellular carcinoma.

\section{Discussion}

We found that patients who were closely followed-up post discharge had numerically fewer HF hospital readmissions. This trend is consistent with previously published literature (Al-Bawardy $\mathrm{R}$ et al) finding lower $\mathrm{HF}$ readmission rates when patients were managed by a pharmacist following HF hospitalization..$^{10}$ In our study, the control group had similar demographics and severity of HF dismissing the theory that only those who were doing well attended their post discharge appointments. The control group 30-day HF readmission rate of $18 \%$ was similar to the high $\mathrm{HF}$ readmission rate at our institution, thus giving credence to the findings reported here. A unique attribute of our BBC was that it served an urban, medically underserved, African American population at highrisk for $\mathrm{HF}$ readmission. Furthermore, our BBC was managed by a pharmacy resident, illustrating the value of utilizing pharmacy trainees in post-discharge heart failure clinics. Due to their expertise, pharmacists are particularly well-suited to provide patients the necessary medication interventions to optimize medication regimen and improve quality of life.

Some limitations to our study should be noted. The sample size was small and likely underpowered to detect a statistical difference. This might be influenced by low socioeconomic status of our patient population. Unlike Al-Bawardy $\mathrm{R}$ et al, whose clinic was established at the Montefiore Medical Center in the Bronx and served a multiracial diverse patient population, the majority of our patients were African American and resided in Detroit. ${ }^{10}$ When compared with the 100 largest cities in the nation, Detroit has the highest poverty rate for residents with 1 in 4 households not owning a car and 
depending on the city's transit system. ${ }^{11}$ Furthermore, African American patients experience a $50 \%$ higher incidence of $\mathrm{HF}$ relative to the general population, as well as an increased risk of mortality secondary to chronic HF as compared with White patients. ${ }^{12}$ With respect to continuity of care, our patients were only followed for a short period of time at our BBC (6 months compared to 2 years at the Montefiore Medical Center) and we hypothesize that the benefits of the BBC could have been greater with a longer period of follow-up. Also, medication adherence was self-reported. While medications were visually reviewed, adherence assessment was still subjective in nature.

\section{Conclusion}

In summary, our study demonstrated that post-discharge follow-up with HF patients by pharmacists was valuable. Our clinic had numerically fewer HF hospital readmissions compared with a scheduled but "no show" control group in a high-risk urban population. Continued efforts should be directed towards increasing the utilization of pharmacists in caring for patients with HF, evaluating the economic and clinical outcomes.

Disclosures: There are no disclosures to make for any of the authors.

Funding: This research did not receive any specific grant from funding agencies in the public, commercial, or not-for-profit sectors.

\section{References}

1. Benjamin EJ, Virani SS, Callaway CW, et al. Heart disease and stroke statistics-2018 update: A report from the American Heart Association. Circulation 2018;137:e67-e492.

2. Christopher MO. High Heart Failure Readmission Rates: Is it the Health System's Fault? JACC 2017;5(5):317-398.

3. Fang J, Mensah GA, Croft JB, Keenan NL. Heart failure-related hospitalization in the U.S., 1979 to 2004. J Am Coll Cardiol. 2008;52: 428-34.

4. Hospital Compare. www.medicare.gov/hospitalcompare.

5. Ankur G, Gregg CF. The Hospital Readmissions Reduction Program. JACC 2018;6(7):607-609.

6. Benjamin EJ, Muntner P, Alonso A, Bittencourt MS, Callaway CW, Carson AP, et al. Heart disease and stroke statistics - 2019 update: a report from the American Heart Association. Circulation. 2019;139(10):e56-528.

7. Jencks SF, Williams MV, Coleman EA. Rehospitalizations among patients in the Medicare fee-for-service program. N Engl J Med. 2009;360:1418-28.

8. Clyde W. Y, et al. 2017 ACC/AHA/HFSA Focused Update of the 2013 ACCF/AHA Guideline for the Management of Heart Failure. Circulation. 2017;136:e137-e161

9. Milfred-Laforest SK, Chow SL, Didomenico RJ, et al. Clinical pharmacy services in heart failure: an opinion paper from the Heart Failure Society of America and American College of Clinical Pharmacy Cardiology Practice and Research Network. J Cardiac Fail 2013;19:354-69.

10. Al-Bawardy R, et al. Heart Failure Post Discharge Clinic: A Pharmacist-led Approach to Reduce Readmissions. Curr Probl Cardiol 2019;44(10):1-14.

11. National Equity Atlas. An Equity Profile of the City of Detroit. 2017.

https://nationalequityatlas.org/sites/default/files/det roit-city-final.pdf

12. Sidney S, Quesenberry C, Jaffe M, et al. Recent trends in cardiovascular mortality in the United States and public health goals. JAMA Cardiol 2016; 1: 594-599 
Table 1: Baseline Characteristics

\begin{tabular}{|c|c|c|c|}
\hline & $\begin{array}{c}\text { BBC: Show } \\
N=15\end{array}$ & $\begin{array}{c}\text { BBC: No Show } \\
N=17\end{array}$ & $P$ value \\
\hline & \multicolumn{3}{|c|}{ Demographics } \\
\hline Age (years), mean \pm SD & $62 \pm 11$ & $59 \pm 10$ & 0.24 \\
\hline Female gender, $\mathrm{n}(\%)$ & $8(53)$ & $10(59)$ & 0.75 \\
\hline \multirow[t]{2}{*}{ African American race, $\mathrm{n}(\%)$} & $13(87)$ & $14(82)$ & 0.74 \\
\hline & \multicolumn{3}{|c|}{ Medical History, n (\%) } \\
\hline HF exacerbations in the last 12 months, $n$ (mean \pm SD) & $34(2 \pm 2)$ & $58(3 \pm 3)$ & 0.10 \\
\hline Patients on infusions during hospital stay & $7(47)$ & $8(47)$ & 0.98 \\
\hline History of interventions (CABG, CABG/pacemakers) & $5(33)$ & $9(53)$ & 0.26 \\
\hline $\begin{array}{l}\text { Left ventricular Ejection Fraction (LVEF) } \\
-E F>40 \% \\
-E F 20-40 \% \\
-E F<20 \%\end{array}$ & $\begin{array}{l}4(27) \\
5(33) \\
6(40)\end{array}$ & $\begin{array}{l}6(35) \\
2(12) \\
9(53)\end{array}$ & $\begin{array}{l}0.60 \\
0.14 \\
0.46 \\
\end{array}$ \\
\hline Atrial fibrillation/Atrial flutter & $3(20)$ & $5(29)$ & 0.54 \\
\hline Diabetes mellitus & $5(33)$ & $8(47)$ & 0.43 \\
\hline Hypertension & $13(87)$ & $17(100)$ & 0.12 \\
\hline Chronic kidney disease & $7(47)$ & $5(29)$ & 0.31 \\
\hline Cancer & $2(13)$ & $4(24)$ & 0.46 \\
\hline Anemia & $12(80)$ & $13(76)$ & 0.81 \\
\hline Obstructive sleep apnea & $2(13)$ & $5(29)$ & 0.27 \\
\hline Current smoker & $6(40)$ & $3(18)$ & 0.16 \\
\hline \multirow[t]{2}{*}{ History of illicit drug abuse } & $4(27)$ & $2(12)$ & 0.28 \\
\hline & \multicolumn{3}{|c|}{ Laboratory Values Upon Discharge, average } \\
\hline $\mathrm{K}(\mathrm{mg} / \mathrm{dL})$ & 4 & 4 & 0.28 \\
\hline BUN (mg/dL) & 22 & 39 & $<0.01$ \\
\hline $\mathrm{SCr}(\mathrm{mg} / \mathrm{dL})$ & 1 & 2 & 0.05 \\
\hline Hemoglobin (g/dL) & 11 & 11 & 0.48 \\
\hline Hematocrit (\%) & 36 & 35 & 0.35 \\
\hline $\mathrm{BNP}(\mathrm{pg} / \mathrm{mL})$ & 2194 & 1437 & 0.25 \\
\hline
\end{tabular}

BBC: brown bag clinic, BNP: b-type natriuretic peptide, BUN: blood urea nitrogen, CABG: coronary artery bypass graft, EF: ejection fraction, HF: heart failure, K: potassium, LVEF: left ventricular ejection fraction, $n$ : number, SCr: serum creatinine, SD: standard deviation 
Table 2: Pharmacists' Interventions

\begin{tabular}{|l|c|}
\hline \multicolumn{1}{|c|}{ Specific Intervention } & Number \\
\hline Addition/discontinuation of HF medication, dose alteration & 32 \\
\hline Addition of potassium binder & 2 \\
\hline Addition of SGLT-2 inhibitor or GLP-1 agonist & 2 \\
\hline Addition of iron supplementation & 1 \\
\hline Removal of contraindicated or unnecessary medications & 2 \\
\hline Non-HF related interventions & 9 \\
\hline Identifying side effects & 1 \\
\hline Identifying drug-drug interactions & 2 \\
\hline Contacting other healthcare providers regarding management of patient care & 2 \\
\hline Submitting prior authorizations & 1 \\
\hline Conducting phone follow-ups between visits & 2 \\
\hline \multicolumn{2}{|c|}{ Total Number of Interventions } \\
\hline
\end{tabular}

GLP-1: glucagon-like peptide-1, HF: heart failure, SGLT-2: sodium-glucose transport protein-2

Table 3: Outcomes

\begin{tabular}{|l|c|c|c|c|}
\hline & $\begin{array}{c}\text { BBC: Show } \\
\mathrm{N}=14+\end{array}$ & $\begin{array}{c}\text { BBC: No Show } \\
\mathrm{N}=17\end{array}$ & $\begin{array}{c}\text { Total } \\
\mathrm{N}=31\end{array}$ & P value \\
\hline $\begin{array}{l}\text { 30-day HF hospital } \\
\text { readmission, } \mathrm{n}(\%)\end{array}$ & $1(7)$ & $3(18)$ & $4(13)$ & 0.12 \\
\hline $\begin{array}{l}\text { 90-day HF hospital } \\
\text { readmission, } \mathrm{n}(\%)\end{array}$ & $3(21)$ & $7(41)$ & $10(32)$ & 0.20 \\
\hline
\end{tabular}

†One BBC participant was excluded from the primary outcome's analysis due to diagnosis of hepatocellular carcinoma 